\title{
A novel kind of the type 2 poly-Fubini polynomials and numbers
}

\author{
Waseem A. Khan
}

Department of Mathematics and Natural Sciences, Prince Mohammad Bin Fahd University, P.O Box 1664, Al Khobar 31952, Saudi Arabia

E-mail: wkhan1@pmu.edu.sa

\begin{abstract}
Motivation by the definition of the type 2 poly-Bernoulli polynomials introduced by Kim-Kim [9], in the present paper, we consider a new class of new generating function for the Fubini polynomials, called the type 2 poly-Fubini polynomials by means of the polyexponential function. Then, we derive some useful relations and properties. We show that the type 2 poly-Fubini polynomials equal a linear combination of the classical of the Fubini polynomials and Stirling numbers of the first kind. In a special case, we give a relation between the type 2 poly-Fubini polynomials and Bernoulli polynomials of order $r$. Moreover, inspired by the definition of the unipoly-Bernoulli polynomials introduced by Kim-Kim [9]. We introduce the type 2 unipoly-Fubini polynomials by means of unipoly function and give multifarious properties including derivative and integral properties. Furthermore, we provide a correlation between the unipoly-Fubini polynomials and the classical Fubini polynomials.
\end{abstract}

2010 Mathematics Subject Classification.: 11B83, 11B37, 05A19.

Keywords: Polyexponential functions, type 2 poly-Fubini polynomials, unipoly functions.

\section{Introduction}

Special polynomials have their origin in the solution of the differential equations (or partial differential equations) under some conditions. Special polynomials can be defined in a various ways such as by generating functions, by recurrence relations, by $p$-adic integrals in the sense of fermionic and bosonic, by degenerate versions, etc.

Kim-Kim have introduced polyexponential function in [9] and its degenerate version in [19-22]. By making use of aforementioned function, they have introduced a new class of some numbers and polynomials. This idea provides a powerfool tool in order to define special numbers and polynomials by making use of polyexponential function. One may see that the notion of polyexponential function form a special class of polynomials because of their great applicability. The importance of these polynomials would be to find applications in analytic number theory, applications in classical analysis and statistics.

The classical Bernoulli $B_{n}(x)$, Euler $E_{n}(x)$ and Genocchi $G_{n}(x)$ polynomial are defined by means of the following generating function as follows

$$
\frac{t}{e^{t}-1} e^{x t}=\sum_{n=0}^{\infty} B_{n}(x) \frac{t^{n}}{n !},|t|<2 \pi, \frac{2}{e^{t}+1} e^{x t}=\sum_{n=0}^{\infty} E_{n}(x) \frac{t^{n}}{n !},|t|<\pi,
$$

and

$$
\frac{2 t}{e^{t}+1} e^{x t}=\sum_{n=0}^{\infty} G_{n}(x) \frac{t^{n}}{n !},|t|<\pi,(\text { see }[4,11,14])
$$

respectively. 
Kargin [19] defined the two-variable Fubini polynomials by the following generating function

$$
\frac{e^{x t}}{1-y\left(e^{t}-1\right)}=\sum_{n=0}^{\infty} F_{n}(x, y) \frac{t^{n}}{n !},(\text { see }[3,7,16,17,18]) .
$$

When $x=0$ in (1.2), the two variable Fubini polynomials $F_{n}(x, y)$ reduce to the usual Fubini polynomials given by

$$
\frac{1}{1-y\left(e^{t}-1\right)}=\sum_{n=0}^{\infty} F_{n}(y) \frac{t^{n}}{n !},(\text { see }[1,2,5]) .
$$

It is easy to see that

$$
F_{n}\left(x,-\frac{1}{2}\right)=E_{n}(x), F_{n}\left(-\frac{1}{2}\right)=E_{n}
$$

and

$$
F_{n}(y)=\sum_{k=0}^{n} S_{2}(n, k) k ! y^{k}
$$

For $y=1$ in (1.3), we get the familiar Fubini numbers $F_{n}(1)=F_{n}$ as follows

$$
\frac{1}{\left.2-e^{t}\right)}=\sum_{n=0}^{\infty} F_{n} \frac{t^{n}}{n !},(\text { see }[1,2]) \text {. }
$$

For more information about the applications of the usual Fubini polynomials and numbers (see $[24,25,28,29,30])$.

Kim-Kim [9] introduced the polyexponential function, as an inverse to the polylogarithm function to be

$$
\operatorname{Ei}_{k}(x)=\sum_{n=1}^{\infty} \frac{x^{n}}{(n-1) ! n^{k}},(k \in \mathbb{Z}) .
$$

For $k=1,(1.6)$ gives

$$
\operatorname{Ei}_{1}(x)=\sum_{n=1}^{\infty} \frac{x^{n}}{n !}=e^{x}-1
$$

For $k \in \mathbb{Z}$, Kim-Kim considered the type 2 poly-Bernoulli polynomials are defined by means of the following generating function

$$
\frac{\operatorname{Ei}_{k}(\log (1+t))}{e^{t}-1} e^{x t}=\sum_{n=0}^{\infty} B_{n}^{(k)}(x) \frac{t^{n}}{n !},(\text { see }[9]) .
$$

In the special case, $x=0, B_{n}^{(k)}=B_{n}^{(k)}(0)$ are called the type 2 poly-Bernoulli numbers. Note that $B_{n}^{(1)}(x)=B_{n}(x)$ are called the ordinary Bernoulli polynomials.

For $k \in \mathbb{Z}$, the polylogaritm function is defined by

$$
\operatorname{Li}_{k}(x)=\sum_{n=1}^{\infty} \frac{x^{n}}{n^{k}},(|x|<1),(\text { see },[26]) .
$$

Note that

$$
\operatorname{Li}_{1}(x)=\sum_{n=1}^{\infty} \frac{x^{n}}{n}=-\log (1-x)
$$


In (2020), Lee et al. [27] introduced the type 2 poly-Euler polynomials are given by

$$
\frac{\operatorname{Ei}_{k}(1+2 t)}{t\left(e^{t}+1\right)} e^{x t}=\sum_{n=0}^{\infty} E_{n}^{(k)}(x) \frac{t^{n}}{n !} .
$$

In the case when $x=0, E_{n}^{(k)}=E_{n}^{(k)}(0)$ are called the type 2 poly-Euler numbers.

The Daehee polynomials are defined by

$$
\frac{\log (1+t)}{t}(1+t)^{x}=\sum_{n=0}^{\infty} D_{n}(x) \frac{t^{n}}{n !},(\text { see }[6,15]) .
$$

When $x=0, D_{n}=D_{n}(0)$ are called the Daehee numbers.

For $n \geq 0$, the Stirling numbers of the first kind are defined by

$$
(x)_{n}=\sum_{l=0}^{n} S_{1}(n, l) x^{l},(\text { see }[4,9]),
$$

where $(x)_{0}=1$, and $(x)_{n}=x(x-1) \cdots(x-n+1),(n \geq 1)$. From (1.13), it is easily to see that

$$
\frac{1}{k !}(\log (1+t))^{k}=\sum_{n=k}^{\infty} S_{1}(n, k) \frac{t^{n}}{n !},(k \geq 0),(\text { see }[4,9]) .
$$

In the inverse expression to (1.13), the Stirling numbers of the second kind are defined by

$$
x^{n}=\sum_{l=0}^{n} S_{2}(n, l)(x)_{l},(\text { see }[10,12,13]) .
$$

From (1.15), it is easily to see that

$$
\frac{1}{k !}\left(e^{t}-1\right)^{k}=\sum_{n=l}^{\infty} S_{2}(n, l) \frac{t^{n}}{n !},(\text { see }[1-30]) .
$$

In this paper, we construct the poly-Fubini polynomials and numbers, called the type 2 poly-Fubini polynomials and numbers by using the polyexponential function and derive several properties on the poly-Fubini polynomials and numbers. In the final section, we define type 2 unipoly-Fubini polynomials by means of unipoly function and derive explicit expressions.

\section{Type 2 poly-Fubini polynomials and numbers}

In this section, we define the type 2 poly-Fubini polynomials using the polyexponential functions and represent the usual Fubini polynomials (more precisely, the values of Fubini polynomials at 1 ) when $k=1$. At the same time, we give explicit expressions and identities involving those polynomials.

For $k \in \mathbb{Z}$, the type 2 poly-Fubini polynomials are defined via the following exponential generating function (in a suitable neighborhood of $t=0$ ) including the polyexponential function as given below:

$$
\frac{\operatorname{Ei}_{k}(\log (1+t))}{t\left(1-y\left(e^{t}-1\right)\right)} e^{x t}=\sum_{n=0}^{\infty} F_{n}^{(k)}(x ; y) \frac{t^{n}}{n !} .
$$


At the point $x=0$ and $y=1, F_{n}^{(k)}=F_{n}^{(k)}(0 ; 1)$ are called the type 2 poly-Fubini numbers.

For $k=1$, by using (1.6) and (2.1), we see that

$$
\frac{\operatorname{Ei}_{1}(\log (1+t))}{t\left(1-y\left(e^{t}-1\right)\right)} e^{x t}=\frac{1}{1-y\left(e^{t}-1\right)} e^{x t}=\sum_{n=0}^{\infty} F_{n}(x) \frac{t^{n}}{n !},
$$

where $F_{n}(x)$ are called the Fubini polynomials (see Eq. (1.3)).

By (2.1) and (2.2), we get

$$
F_{n}^{(1)}(x)=F_{n}(x),(n \geq 0) .
$$

Using (1.6), we see that

$$
\begin{gathered}
\operatorname{Ei}_{k}(\log (1+t))=\sum_{m=1}^{\infty} \frac{(\log (1+t))^{m}}{(m-1) ! m^{k}} \\
=\sum_{m=0}^{\infty} \frac{(\log (1+t))^{m+1}}{m !(m+1)^{k}} \\
=\sum_{m=0}^{\infty} \frac{1}{(m+1)^{k-1}} \frac{1}{(m+1) !}(\log (1+t))^{m+1} \\
=\sum_{m=0}^{\infty} \frac{1}{(m+1)^{k-1}} \sum_{l=m+1}^{\infty} S_{1}(l, m+1) \frac{t^{l}}{l !} .
\end{gathered}
$$

From (2.3) and (2.1), we have

$$
\begin{gathered}
\frac{e^{x t}}{t\left(1-y\left(e^{t}-1\right)\right)} \operatorname{Ei}_{k}(\log (1+t))=\frac{1}{1-y\left(e^{t}-1\right)} e^{x t} \sum_{m=0}^{\infty} \frac{1}{(m+1)^{k-1}} \sum_{l=m}^{\infty} \frac{S_{1}(l+1, m+1)}{l+1} \frac{t^{l}}{l !} \\
=\sum_{n=0}^{\infty} F_{n}(x ; y) \frac{t^{n}}{n !} \sum_{m=0}^{\infty} \frac{1}{(m+1)^{k-1}} \sum_{l=m}^{\infty} \frac{S_{1}(l+1, m+1)}{l+1} \frac{t^{l}}{l !} \\
\text { L.H.S }=\sum_{n=0}^{\infty}\left(\sum_{l=0}^{n}\left(\begin{array}{l}
n \\
l
\end{array}\right) \sum_{m=0}^{l} \frac{1}{(m+1)^{k-1}} \frac{S_{1}(l+1, m+1)(1-u)^{l}}{l+1} F_{n-l}(x ; y)\right) \frac{t^{n}}{n !} .
\end{gathered}
$$

Therefore, by (2.3) and (2.4), we obtain the following theorem.

Theorem 2.1. For $k \in \mathbb{Z}$ and $n \geq 0$, we have

$$
F_{n}^{(k)}(x ; y)=\sum_{l=0}^{n}\left(\begin{array}{l}
n \\
l
\end{array}\right) \sum_{m=0}^{l} \frac{1}{(m+1)^{k-1}} \frac{S_{1}(l+1, m+1)(1-u)^{l}}{l+1} F_{n-l}(x ; y) .
$$

Corollary 2.1. For $n \geq 0$, we have

$$
F_{n}(x ; y)=\sum_{l=0}^{n}\left(\begin{array}{l}
n \\
l
\end{array}\right) \sum_{m=0}^{l} \frac{S_{1}(l+1, m+1)}{l+1} F_{n-l}(x ; y) .
$$

Moreover,

$$
\sum_{l=1}^{n}\left(\begin{array}{l}
n \\
l
\end{array}\right) \sum_{m=0}^{l} \frac{S_{1}(l+1, m+1) 2^{l}}{l+1} F_{n-l}(x ; y)=0,(n \geq 1) .
$$

It is well-known from $([8,11])$ that 


$$
\left(\frac{t}{\log (1+t)}\right)^{r}(1+t)^{x-1}=\sum_{n=0}^{\infty} B_{n}^{(n-r+1)}(x) \frac{t^{n}}{n !},(r \in \mathbb{C})
$$

where $B_{n}^{(r)}(x)$ are called the higher-order Bernoulli polynomials which are given by the generating function

$$
\left(\frac{t}{e^{t}-1}\right)^{r} e^{x t}=\sum_{n=0}^{\infty} B_{n}^{(r)}(x) \frac{t^{n}}{n !}
$$

Using (1.6), we have

$$
\begin{aligned}
\frac{d}{d x} \operatorname{Ei}_{k}(\log (1+x)) & =\frac{d}{d x} \sum_{n=1}^{\infty} \frac{(\log (1+x))^{n}}{(n-1) ! n^{k}} \\
& =\frac{1}{1+x} \sum_{n=1}^{\infty} \frac{n(\log (1+x))^{n-1}}{(n-1) ! n^{k}} \\
= & \frac{1}{(1+x) \log (1+x)} \sum_{n=1}^{\infty} \frac{(\log (1+x))^{n}}{(n-1) ! n^{k-1}} \\
= & \frac{1}{(1+x) \log (1+x)} \operatorname{Ei}_{k-1}(\log (1+x)
\end{aligned}
$$

From (2.6), $k \geq 1$, we have

$$
\begin{gathered}
\operatorname{Ei}_{k}(\log (1+x))=\int_{0}^{x} \frac{1}{(1+t) \log (1+t)} \\
\times \int_{0}^{t} \underbrace{\frac{1}{(1+t) \log (1+t)} \cdots \int_{0}^{t} \frac{t}{(1+t) \log (1+t)} d t d t \ldots d t .}_{k-2-\text { times }} \\
=x \sum_{m=0}^{\infty} \sum_{m_{1}+\cdots+m_{k-1}=m}\left(\begin{array}{c}
m \\
m_{1}, \cdots, m_{k-1}
\end{array}\right) \frac{B_{m_{1}}^{\left(m_{1}\right)}}{m_{1}+1} \frac{B_{m_{2}}^{\left(m_{2}\right)}}{m_{1}+m_{2}+1} \cdots \frac{B_{m_{k-1}}^{\left(m_{k-1}\right)}}{m_{1}+\cdots+m_{k-1}+1} \frac{x^{m}}{m !}
\end{gathered}
$$

From (2.7), we observe that

$$
\begin{aligned}
& \sum_{n=0}^{\infty} F_{n}^{(k)}(y) \frac{x^{n}}{n !}=\frac{1}{x\left(1-y\left(e^{x}-1\right)\right)} \operatorname{Ei}_{k}(\log (1+x)) \\
&=\frac{x}{x\left(1-y\left(e^{x}-1\right)\right)} \\
& \times \sum_{m=0}^{\infty} \sum_{m_{1}+\cdots+m_{k-1}=m}\left(\begin{array}{c}
m \\
m_{1}, \cdots, m_{k-1}
\end{array}\right) \frac{B_{m_{1}}^{\left(m_{1}\right)}}{m_{1}+1} \frac{B_{m_{2}}^{\left(m_{2}\right)}}{m_{1}+m_{2}+1} \cdots \frac{B_{m_{k-1}}^{\left(m_{k-1}\right)}}{m_{1}+\cdots+m_{k-1}+1} \frac{x^{m}}{m !} \\
&=\sum_{n=0}^{\infty} \sum_{m=0}^{n}\left(\begin{array}{c}
n \\
m
\end{array}\right) \sum_{m=0}^{\infty} \\
& \quad \sum_{m_{1}+\cdots+m_{k-1}=m}\left(\begin{array}{c}
B_{m_{1}}^{\left(m_{1}\right)} \\
m_{1}, \cdots, m_{k-1}
\end{array}\right) \frac{B_{m_{2}}^{\left(m_{2}\right)}}{m_{1}+1} \frac{B_{1}+m_{2}+1}{m_{m_{k-1}}} \cdots \frac{B_{1}+\cdots+m_{k-1}+1}{m_{n-m}(y) \frac{x^{n}}{n !}} .
\end{aligned}
$$

Therefore, by (2.7) and (2.8), we get the following theorem. 
Theorem 2.2. For $n \geq 0$, we have

$$
\begin{gathered}
F_{n}^{(k)}(y)=\sum_{m=0}^{n}\left(\begin{array}{c}
n \\
m
\end{array}\right) \sum_{m=0}^{\infty} \\
\times \sum_{m_{1}+\cdots+m_{k-1}=m}\left(\begin{array}{c}
m \\
m_{1}, \cdots, m_{k-1}
\end{array}\right) \frac{B_{m_{1}}^{\left(m_{1}\right)}}{m_{1}+1} \frac{B_{m_{2}}^{\left(m_{2}\right)}}{m_{1}+m_{2}+1} \cdots \frac{B_{m_{k-1}}^{\left(m_{k-1}\right)}}{m_{1}+\cdots+m_{k-1}+1} F_{n-m}(y) .
\end{gathered}
$$

Corollary 2.2. For $n \geq 0$, we have

$$
F_{n}^{(2)}(y)=\sum_{m=0}^{n}\left(\begin{array}{c}
n \\
m
\end{array}\right) \frac{B_{m}^{m}}{m+1} F_{n-l} .
$$

Let $s \in \mathbb{C}$ and $k \in \mathbb{Z}$ with $k \geq 1$. We consider the function $\eta_{k}$ by the representation of improper integral as follows

$$
\eta_{k}(s)=\frac{1}{\Gamma(s)} \int_{0}^{\infty} \frac{t^{s-1}}{t\left(1-y\left(e^{t}-1\right)\right)} \operatorname{Ei}_{k}(\log (1+t)) d t
$$

We observe that $\eta_{k}(s)$ is holomorphic function for $\Re(s)>0$ because of the comparison test as $\operatorname{Ei}_{k}(\log (1+2 t)) \leq \operatorname{Ei}_{1}(\log (1+2 t))$ with the assumption $2 t \geq 0$. By (2.9), we have

$\eta_{k}(s)=\frac{1}{\Gamma(s)} \int_{0}^{1} \frac{t^{s-1}}{t\left(1-y\left(e^{t}-1\right)\right)} \operatorname{Ei}_{k}(\log (1+t)) d t+\frac{1}{\Gamma(s)} \int_{1}^{\infty} \frac{t^{s-1}}{t\left(1-y\left(e^{t}-1\right)\right)} \operatorname{Ei}_{k}(\log (1+t)) d t$.

The second integral in (2.10) converges absolutely for any $s \in \mathbb{C}$ and thus, the second term on the right hand side vanishes at non positive integers. Hence, we get

$$
\lim _{s \rightarrow-m}\left|\frac{1}{\Gamma(s)} \int_{0}^{1} \frac{t^{s-1}}{t\left(1-y\left(e^{t}-1\right)\right)} \operatorname{Ei}_{k}(\log (1+t)) d t\right| \leq \frac{1}{\Gamma(-m)} M=0
$$

since

$$
\Gamma(s) \Gamma(1-s)=\frac{\pi}{\sin (\pi s)}
$$

Moreover, for $\Re(s)>0$, the first integral in (2.10) can be written as

$$
\begin{gathered}
\frac{1}{\Gamma(s)} \int_{0}^{1} \frac{t^{s-1}}{t\left(1-y\left(e^{t}-1\right)\right)} \operatorname{Ei}_{k}(\log (1+t)) d t=\frac{1}{\Gamma(s)} \sum_{n=0}^{\infty} \frac{F_{n}^{(k)}(y)}{n !} \int_{0}^{1} t^{n+s-1} d t \\
=\frac{1}{\Gamma(s)} \sum_{n=0}^{\infty} \frac{F_{n}^{(k)}(y)}{n !} \frac{1}{n+s}
\end{gathered}
$$

which defines an entire function of $s$. Hence, we arrive that $\eta_{k}(s)$ can be continued to an entire function of $s$. From (2.10) and (2.11), we obtain

$$
\begin{gathered}
\eta_{k}(-m)=\lim _{s \rightarrow-m} \frac{1}{\Gamma(s)} \int_{0}^{1} \frac{t^{s-1}}{t\left(1-y\left(e^{t}-1\right)\right)} \operatorname{Ei}_{k}(\log (1+t)) d t \\
=\lim _{s \rightarrow-m} \frac{1}{\Gamma(s)} \sum_{n=0}^{\infty} \frac{F_{n}^{(k)}(y)}{n !(n+s)} \\
=\cdots+\cdots+0+\lim _{s \rightarrow-m} \frac{1}{\Gamma(s)} \frac{F_{m}^{(k)}}{m !(m+s)}+0+0+\cdots \\
=\lim _{s \rightarrow-m} \frac{1}{m+s} \frac{\Gamma(1-s) \sin (\pi s)}{\pi} \frac{F_{m}^{(k)}(y)}{m !} \\
=\Gamma(1+m) \cos (\pi m) \frac{F_{m}^{(k)}(y)}{m !}
\end{gathered}
$$




$$
=(-1)^{m} F_{m}^{(k)}(y)
$$

Thus, we get the following theorem.

Theorem 2.3. Let $k \in \mathbb{N}$. The function $\eta_{k}(s)$ has an analytic continuation to an function of $s \in \mathbb{C}$, and the special values at non positive integers are given by

$$
\eta_{k}(-m)=(-1)^{m} F_{m}^{(k)}(y),\left(m \in \mathbb{N}_{0}\right) .
$$

From (2.1), we have

$$
\begin{gathered}
\sum_{n=0}^{\infty} F_{n}^{(k)}(x+z ; y) \frac{t^{n}}{n !}=\left(\frac{\operatorname{Ei}_{k}(\log (1+t))}{t\left(1-y\left(e^{t}-1\right)\right)}\right) e^{(x+z) t} \\
=\left(\sum_{n=0}^{\infty} F_{n}^{(k)}(x ; y) \frac{t^{n}}{n !}\right)\left(\sum_{m=0}^{\infty} z^{m} \frac{t^{m}}{m !}\right) \\
\text { L.H.S }=\sum_{n=0}^{\infty}\left(\sum_{m=0}^{n}\left(\begin{array}{c}
n \\
m
\end{array}\right) F_{n-m}^{(k)}(x ; y) z^{m}\right) \frac{t^{n}}{n !}
\end{gathered}
$$

By comparing the coefficients of $t^{n}$, we obtain the following theorem.

Theorem 2.4. For $n \geq 0$, we have

$$
F_{n}^{(k)}(x+z ; y)=\sum_{m=0}^{n}\left(\begin{array}{l}
n \\
m
\end{array}\right) F_{n-m}^{(k)}(x ; y) z^{m} .
$$

From (2.1), we note that

$$
\begin{gathered}
\frac{\operatorname{Ei}_{k}(\log (1+t))}{t}=\left(1-y\left(e^{t}-1\right)\right) \sum_{n=0}^{\infty} F_{n}^{(k)}(y) \frac{t^{n}}{n !} \\
=\sum_{n=0}^{\infty} F_{n}^{(k)}(y) \frac{t^{n}}{n !}-y \sum_{n=0}^{\infty} \sum_{m=0}^{n}\left(\begin{array}{c}
n \\
m
\end{array}\right) F_{n-m}^{(k)}(y) \frac{t^{n}}{n !}+y \sum_{n=0}^{\infty} F_{n}^{(k)}(y) \frac{t^{n}}{n !} \\
=\sum_{n=0}^{\infty}\left(F_{n}^{(k)}(y)-y \sum_{m=0}^{n}\left(\begin{array}{c}
n \\
m
\end{array}\right) F_{n-m}^{(k)}(y)+y F_{n}^{(k)}(y)\right) \frac{t^{n}}{n !} \\
=\sum_{n=0}^{\infty}\left((1+y) F_{n}^{(k)}(y)-y \sum_{m=0}^{n}\left(\begin{array}{c}
n \\
m
\end{array}\right) F_{n-m}^{(k)}(y)\right) \frac{t^{n}}{n !} .
\end{gathered}
$$

On the other hand,

$$
\begin{gathered}
\operatorname{Ei}_{k}(\log (1+t))=\frac{1}{t} \sum_{m=1}^{\infty} \frac{(\log (1+t))^{m}}{(m-1) ! m^{k}} \\
=\frac{1}{t} \sum_{m=0}^{\infty} \frac{(\log (1+t))^{m+1}}{m !(m+1)^{k}}=\frac{1}{t} \sum_{m=0}^{\infty} \frac{(\log (1+t))^{m+1}}{(m+1)^{k-1}} \frac{1}{(m+1) !} \\
=\frac{1}{t} \sum_{m=0}^{\infty} \frac{1}{(m+1)^{k-1}} \sum_{n=m+1}^{\infty} S_{1}(n, m+1) \frac{t^{n}}{n !} \\
=\sum_{n=0}^{\infty} \sum_{m=0}^{n} \frac{1}{(m+1)^{k-1}} \frac{S_{1}(n+1, m+1)}{n+1} \frac{t^{n}}{n !} .
\end{gathered}
$$

Therefore, by (2.14) and (2.15), we obtain the following theorem. 
Theorem 2.5. For $n \geq 0$, we have

$F_{n}^{(k)}(y)=\frac{1}{1+y}\left[y \sum_{m=0}^{n}\left(\begin{array}{c}n \\ m\end{array}\right) F_{n-m}^{(k)}(y)+\sum_{m=0}^{n} \frac{1}{(m+1)^{k-1}} \frac{S_{1}(n+1, m+1)}{n+1}\right]$.

By (2.1), we observe that

$$
\begin{aligned}
\sum_{n=0}^{\infty}\left[F_{n}^{(k)}(x+1 ; y)-F_{n}^{(k)}(x ; y)\right] \frac{t^{n}}{n !}=\frac{\operatorname{Ei}_{k}(\log (1+t))}{t\left(1-y\left(e^{t}-1\right)\right)} e^{x t}\left(e^{t}-1\right) & =\frac{\operatorname{Ei}_{k}(\log (1+t))}{t} \frac{e^{x t}}{1-y\left(e^{t}-1\right)}\left(e^{t}-1\right) \\
= & \frac{\operatorname{Ei}_{k}(\log (1+t))}{t} \frac{1}{y}\left[\frac{e^{x t}}{1-y\left(e^{t}-1\right)}-e^{x t}\right] \\
= & \frac{1}{y}\left[\frac{\operatorname{Ei}_{k}(\log (1+t))}{t\left(1-y\left(e^{t}-1\right)\right)} e^{x t}-\frac{\operatorname{Ei}_{k}(\log (1+t))}{t} e^{x t}\right] \\
=\frac{1}{y}\left[\sum_{n=0}^{\infty} F_{n}^{(k)}(x ; y)\right. & \left.-\sum_{l=0}^{n} \sum_{m=0}^{l}\left(\begin{array}{l}
n \\
l
\end{array}\right) \frac{1}{(m+1)^{k-1}} \frac{S_{1}(l+1, m+1)}{l+1} x^{n-l}\right] \frac{t^{n}}{n !} .
\end{aligned}
$$

On comparing the coefficients of $t^{n}$ on both sides, we get the following theorem.

Theorem 2.6. For $n \geq 0$, we have

$$
y F_{n}^{(k)}(x+1 ; y)=(1+y) F_{n}^{(k)}(x ; y)-\sum_{l=0}^{n} \sum_{m=0}^{l}\left(\begin{array}{l}
n \\
l
\end{array}\right) \frac{1}{(m+1)^{k-1}} \frac{S_{1}(l+1, m+1)}{l+1} x^{n-l} .
$$

From (2.1), we note that

$$
\begin{gathered}
\sum_{n=0}^{\infty} \sum_{m=0}^{\infty} F_{n}^{(k)}\left(x_{1} ; y_{1}\right) F_{m}^{(k)}\left(x_{2} ; y_{2}\right) \frac{t^{n}}{n !} \frac{t^{m}}{m !} \\
=\frac{\operatorname{Ei}_{k}(\log (1+t))}{t\left(1-y_{1}\left(e^{t}-1\right)\right)} e^{x_{1} t} \frac{\operatorname{Ei}_{k}(\log (1+t))}{t\left(1-y_{2}\left(e^{t}-1\right)\right)} e^{x_{2} t} \\
=\frac{\left[\operatorname{Ei}_{k}(\log (1+t))\right]^{2}}{t^{2}}\left(\frac{e^{x_{1} t}}{1-y_{1}\left(e^{t}-1\right)} \frac{e^{x_{2} t}}{1-y_{2}\left(e^{t}-1\right)}\right) \\
=\frac{\left[\operatorname{Ei}_{k}(\log (1+t))\right]^{2}}{t^{2}}\left(\frac{y_{2}}{y_{2}-y_{1}} \frac{e^{\left(x_{1}+x_{2}\right) t}}{1-y_{2}\left(e^{t}-1\right)}-\frac{y_{1}}{y_{2}-y_{1}} \frac{e^{\left(x_{1}+x_{2}\right) t}}{1-y_{1}\left(e^{t}-1\right)}\right) \\
=\left(\frac{\sum_{n=0}^{\infty}\left[y_{2} F_{n}^{(k)}\left(x_{1}+x_{2} ; y_{2}\right)-y_{1} F_{n}^{(k)}\left(x_{1}+x_{2} ; y_{1}\right)\right]}{n !}\right) t^{n} .
\end{gathered}
$$

On the other hand,

$$
\begin{gathered}
\sum_{n=0}^{\infty} \sum_{m=0}^{\infty} F_{n}^{(k)}\left(x_{1} ; y_{1}\right) F_{m}^{(k)}\left(x_{2} ; y_{2}\right) \frac{t^{n}}{n !} \frac{t^{m}}{m !} \\
=\sum_{n=0}^{\infty}\left(\sum_{m=0}^{n}\left(\begin{array}{c}
n \\
m
\end{array}\right) F_{n-m}^{(k)}\left(x_{1} ; y_{1}\right) F_{m}^{(k)}\left(x_{2} ; y_{2}\right)\right) \frac{t^{n}}{n !} .
\end{gathered}
$$

Therefore, by (2.17) and (2.18), we obtain the following theorem.

Theorem 2.7. For $n \geq 0$, we have

$$
\sum_{m=0}^{n}\left(\begin{array}{c}
n \\
m
\end{array}\right) F_{n-m}^{(k)}\left(x_{1} ; y_{1}\right) F_{m}^{(k)}\left(x_{2} ; y_{2}\right)
$$


Using (2.1), we have

$$
=\frac{y_{2} F_{n}^{(k)}\left(x_{1}+x_{2} ; y_{2}\right)-y_{1} F_{n}^{(k)}\left(x_{1}+x_{2} ; y_{1}\right)}{y_{2}-y_{1}} .
$$

$$
\begin{gathered}
\sum_{n=0}^{\infty} F_{n}^{(k)}(y) \frac{t^{n}}{n !}=\frac{\operatorname{Ei}_{k}(\log (1+t))}{t\left(1-y\left(e^{t}-1\right)\right)} \\
=\frac{\operatorname{Ei}_{k}(\log (1+t))}{t} \sum_{m=0}^{\infty} y^{m} \sum_{l=m}^{\infty} m ! S_{2}(l, m) \frac{t^{l}}{l !} \\
=\left(\sum_{n=0}^{\infty} \sum_{m=0}^{n} \frac{1}{(m+1)^{k-1}} \frac{S_{1}(n+1, m+1)}{n+1} \frac{t^{n}}{n !}\right)\left(\sum_{l=0}^{\infty} \sum_{r=0}^{l} y^{r} r ! S_{2}(l, r) \frac{t^{l}}{l !}\right) \\
=\sum_{n=0}^{\infty}\left(\sum_{l=0}^{n} \sum_{m=0}^{n-l} \sum_{r=0}^{l}\left(\begin{array}{l}
n \\
l
\end{array}\right) \frac{1}{(m+1)^{k-1}} \frac{S_{1}(n-l+1, m+1)}{n-l+1} y^{r} r ! S_{2}(l, r)\right) .
\end{gathered}
$$

Therefore, by (2.19), we obtain the following theorem.

Theorem 2.8. For $n \geq 0$, we have

$$
F_{n}^{(k)}(y)=\sum_{l=0}^{n} \sum_{m=0}^{n-l} \sum_{r=0}^{l}\left(\begin{array}{l}
n \\
l
\end{array}\right) \frac{1}{(m+1)^{k-1}} \frac{S_{1}(n-l+1, m+1)}{n-l+1} y^{r} r ! S_{2}(l, r) .
$$

From (2.1), we observe that

$$
\frac{\operatorname{Ei}_{k}(\log (1+t))}{t\left(1-y\left(e^{t}-1\right)\right)}=\sum_{n=0}^{\infty} F_{n}^{(k)}(y) \frac{t^{n}}{n !} .
$$

Replacing $t$ by $e^{t}-1$, we get

$$
\begin{aligned}
& \sum_{m=0}^{\infty} F_{m}^{(k)}(y) \frac{\left(e^{t}-1\right)^{m}}{m !}=\frac{\operatorname{Ei}_{k}(t)}{\left(e^{t}-1\right)\left(1-y\left(e^{e^{t}-1}-1\right)\right)} \\
& =\frac{1}{1-y\left(e^{e^{t}-1}-1\right)} \frac{t}{e^{t}-1} \frac{\operatorname{Ei}_{k}(t)}{t} \\
& =\sum_{p=0}^{\infty} F_{p}(y) \frac{\left(e^{t}-1\right)^{p}}{p !} \sum_{q=0}^{\infty} B_{n} \frac{t^{q}}{q !} \sum_{n=0}^{\infty} \frac{t^{n}}{n !(n+1)^{k}} \\
& =\sum_{p=0}^{\infty} F_{p}(y) \sum_{p=i}^{\infty} S_{1}(i, p) \frac{t^{i}}{i !} \sum_{q=0}^{\infty} B_{q} \frac{t^{q}}{q !} \sum_{n=0}^{\infty} \frac{t^{n}}{n !(n+1)^{k}} \\
& =\sum_{i=0}^{\infty} \sum_{p=0}^{i} F_{p}(y) S_{1}(i, p) \frac{t^{i}}{i !} \sum_{q=0}^{\infty} B_{q} \frac{t^{q}}{q !} \sum_{n=0}^{\infty} \frac{t^{n}}{n !(n+1)^{k}} \\
& =\sum_{q=0}^{\infty}\left(\sum_{i=0}^{q} \sum_{p=0}^{i}\left(\begin{array}{l}
q \\
i
\end{array}\right) F_{p}(y) S_{1}(i, p) B_{q-i}\right) \sum_{n=0}^{\infty} \frac{t^{n}}{n !(n+1)^{k}} \\
& =\sum_{n=0}^{\infty}\left(\sum_{q=0}^{n} \sum_{i=0}^{q} \sum_{p=0}^{i}\left(\begin{array}{l}
q \\
i
\end{array}\right)\left(\begin{array}{l}
n \\
q
\end{array}\right) F_{p}(y) S_{1}(i, p) B_{q-i} \frac{1}{(n-q+1)^{k}}\right) \frac{t^{n}}{n !} .
\end{aligned}
$$

On the other hand,

$$
\sum_{m=0}^{\infty} F_{m}^{(k)}(y) \frac{\left(e^{t}-1\right)^{m}}{m !}
$$




$$
\begin{aligned}
& =\sum_{m=0}^{\infty} F_{m}^{(k)}(y) \sum_{n=m}^{\infty} S_{1}(n, m) \frac{t^{n}}{n !} \\
& \sum_{n=0}^{\infty}\left(\sum_{m=0}^{n} F_{m}^{(k)}(y) S_{1}(n, m)\right) \frac{t^{n}}{n !} .
\end{aligned}
$$

Therefore, by (2.20) and (2.21), we get the following theorem.

Theorem 2.9. For $n \geq 0$, we have

$$
\sum_{m=0}^{n} F_{m}^{(k)}(y) S_{1}(n, m)=\sum_{q=0}^{n} \sum_{i=0}^{q} \sum_{p=0}^{i}\left(\begin{array}{c}
q \\
i
\end{array}\right)\left(\begin{array}{l}
n \\
q
\end{array}\right) F_{p}(y) S_{1}(i, p) B_{q-i} \frac{1}{(n-q+1)^{k}} .
$$

\section{The type 2 unipoly-Fubini polynomials and numbers}

In this section, we consider the type 2 unipoly-Fubini polynomials and numbers and investigate some properties of those polynomials.

Let $p$ be any arithmetic function which is a real or complex valued function defined on the set of positive integers $\mathbb{N}$. Kim-Kim [9] defined the unipoly function attached to polynomials $p(x)$ by

$$
u_{k}(x \mid p)=\sum_{n=1}^{\infty} \frac{p(n)}{n^{k}} x^{n},(k \in \mathbb{Z}) .
$$

Moreover,

$$
u_{k}(x \mid 1)=\sum_{n=1}^{\infty} \frac{x^{n}}{n^{k}}=\operatorname{Li}_{k}(x),(\text { see }[26]),
$$

is the ordinary polylogaritm function. $p(x)$ by

Now, we define the type 2 unipoly-Fubini polynomials attached to polynomials

$$
\frac{1}{t\left(1-y\left(e^{t}-1\right)\right)} u_{k}(\log (1+t) \mid p) e^{x t}=\sum_{n=0}^{\infty} F_{n, p}^{(k)}(x ; y) \frac{t^{n}}{n !} .
$$

When $x=0$ and $y=1, F_{n, p}^{(k)}=F_{n, p}^{(k)}(0 ; 1)$ are called the unipoly-Fubini numbers attached to $p$.

If we take $p(n)=\frac{1}{\Gamma(n)}$. Then, we have

$$
\begin{gathered}
\sum_{n=0}^{\infty} F_{n, \frac{1}{\Gamma}}^{(k)}(x ; y) \frac{t^{n}}{n !}=\frac{1}{t\left(1-y\left(e^{t}-1\right)\right)} e^{x t} u_{k}\left(\log (1+t) \mid \frac{1}{\Gamma}\right) \\
=\frac{1}{t\left(1-y\left(e^{t}-1\right)\right)} e^{x t} \sum_{m=1}^{\infty} \frac{(\log (1+t))^{m}}{m^{k}(m-1) !} .
\end{gathered}
$$

In particular, for $k=1$, we obtain

$$
\sum_{n=0}^{\infty} F_{n, \frac{1}{\Gamma}}^{(1)}(x ; y) \frac{t^{n}}{n !}=\frac{1}{t\left(1-y\left(e^{t}-1\right)\right)} e^{x t} \sum_{m=1}^{\infty} \frac{(\log (1+t))^{m}}{m !}=\frac{1}{1-y\left(e^{t}-1\right)} e^{x t}
$$

Therefore by (3.3) and (3.5), we have

$$
F_{n, \frac{1}{\Gamma}}^{(k)}(x ; y)=F_{n}(x ; y) .
$$


From (3.3), we have

$$
\begin{gathered}
\sum_{n=0}^{\infty} F_{n, p}^{(k)}(x ; y) \frac{t^{n}}{n !}=\frac{1}{t\left(1-y\left(e^{t}-1\right)\right)} e^{x t} u_{k}(\log (1+t) \mid p) \\
=\frac{1}{t\left(1-y\left(e^{t}-1\right)\right)} e^{x t} \sum_{m=1}^{\infty} \frac{p(m)}{m^{k}}(\log (1+t))^{m} \\
=\frac{1}{t\left(1-y\left(e^{t}-1\right)\right)} e^{x t} \sum_{m=0}^{\infty} \frac{p(m+1)}{(m+1)^{k}}(\log (1+t))^{m+1} \\
=\frac{1}{t\left(1-y\left(e^{t}-1\right)\right)} e^{x t} \sum_{m=0}^{\infty} \frac{p(m+1)(m+1) !}{(m+1)^{k}} \sum_{l=m+1}^{\infty} S_{1}(l, m+1) \frac{t^{l}}{l !} \\
=\frac{1}{1-y\left(e^{t}-1\right)} e^{x t} \sum_{m=0}^{\infty} \frac{p(m+1)(m+1) !}{(m+1)^{k}} \sum_{l=m}^{\infty} \frac{S_{1}(l+1, m+1)}{l+1} \frac{t^{l}}{l !} \\
=\sum_{n=0}^{\infty} F_{n}(x ; y) \frac{t^{n}}{n !} \sum_{l=0}^{\infty}\left(\sum_{m=0}^{l} \frac{p(m+1)(m+1) !}{(m+1)^{k}} \frac{S_{1}(l+1, m+1)}{l+1}\right) \frac{t^{l}}{l !} \\
\text { L.H.S = } \sum_{n=0}^{\infty}\left(\sum_{l=0}^{n} \sum_{m=0}^{l}\left(\begin{array}{l}
n \\
l
\end{array}\right) \frac{p(m+1)(m+1) !}{(m+1)^{k}(l+1, m+1)}\right.
\end{gathered}
$$

Therefore, by comparing the coefficients on both sides of (3.7), we obtain the following theorem.

Theorem 3.1. For $n \geq 0$ and $k \in \mathbb{Z}$, we have

$$
F_{n, p}^{(k)}(x ; y)=\sum_{l=0}^{n} \sum_{m=0}^{l}\left(\begin{array}{l}
n \\
l
\end{array}\right) \frac{p(m+1)(m+1) !}{(m+1)^{k}} \frac{S_{1, \lambda}(l+1, m+1)}{l+1} F_{n-l}(x ; y) .
$$

Moreover,

$$
F_{n, \frac{1}{\Gamma}}^{(k)}(x ; y)=\sum_{l=0}^{n} \sum_{m=0}^{l}\left(\begin{array}{l}
n \\
l
\end{array}\right) \frac{1}{(m+1)^{k-1}} \frac{S_{1}(l+1, m+1)}{l+1} F_{n-l}(x ; y) .
$$

From (3.3), we have

$$
\begin{gathered}
\sum_{n=0}^{\infty} F_{n, p}^{(k)}(x ; y) \frac{t^{n}}{n !}=\frac{1}{t\left(1-y\left(e^{t}-1\right)\right)} u_{k}(\log (1+t) \mid p) e^{x t} \\
=\frac{1}{t\left(1-y\left(e^{t}-1\right)\right)} u_{k}(\log (1+t) \mid p)\left(e^{t}-1+1\right)^{x} \\
=\frac{1}{t\left(1-y\left(e^{t}-1\right)\right)} u_{k}(\log (1+t) \mid p) \sum_{m=0}^{\infty}(x)_{m} \frac{\left(e^{t}-1\right)^{m}}{m !} \\
=\sum_{n=0}^{\infty} F_{n, p}^{(k)}(y) \frac{t^{n}}{n !} \sum_{l=0}^{\infty} \sum_{m=0}^{l}(x)_{m} S_{2}(l, m) \frac{t^{l}}{l !} \\
\text { L.H.S }=\sum_{n=0}^{\infty}\left(\sum_{l=0}^{n} \sum_{m=0}^{l}\left(\begin{array}{l}
n \\
l
\end{array}\right)(x)_{m} S_{2}(l, m) F_{n-l, p}^{(k)}(y)\right) \frac{t^{n}}{n !} .
\end{gathered}
$$

By comparing the coefficients on both sides of (3.8), we get the following theorem. 
Theorem 3.2. For $n \geq 0$ and $k \in \mathbb{Z}$, we have

$$
F_{n, p}^{(k)}(x ; y)=\sum_{l=0}^{n} \sum_{m=0}^{l}\left(\begin{array}{l}
n \\
l
\end{array}\right)(x)_{m} S_{2}(l, m) F_{n-l, p}^{(k)}(y) .
$$

From (3.3), we have

$$
\begin{gathered}
\sum_{n=0}^{\infty} F_{n, p}^{(k)}(y) \frac{t^{n}}{n !}=\frac{1}{t\left(1-y\left(e^{t}-1\right)\right)} u_{k}(\log (1+t) \mid p) \\
=\frac{1}{t\left(1-y\left(e^{t}-1\right)\right)} \sum_{m=1}^{\infty} \frac{p(m)}{m^{k}}(\log (1+t))^{m} \\
=\frac{1}{t\left(1-y\left(e^{t}-1\right)\right)} \sum_{m=0}^{\infty} \frac{p(m+1)}{(m+1)^{k}}(\log (1+t))^{m+1} \\
=\frac{\log (1+t)}{t} \frac{1}{1-y\left(e^{t}-1\right)} \sum_{m=0}^{\infty} \frac{p(m+1) m !}{(m+1)^{k}} \sum_{l=m}^{\infty} S_{1}(l, m) \frac{t^{l}}{l !} \\
=\sum_{r=0}^{\infty} D_{r} \frac{t^{r}}{r !} \sum_{n=0}^{\infty} F_{n}(y) \frac{t^{n}}{n !} \sum_{l=0}^{\infty}\left(\sum_{m=0}^{l} \frac{p(m+1) m !}{(m+1)^{k}} S_{1}(l, m) 2^{l}\right) \frac{t^{l}}{l !} \\
=\sum_{n=0}^{\infty} \sum_{r=0}^{n}\left(\begin{array}{l}
n \\
r
\end{array}\right) D_{r} F_{n-r}(y) \frac{t^{n}}{n !} \sum_{l=0}^{\infty}\left(\sum_{m=0}^{l} \frac{p(m+1) m !}{(m+1)^{k}} S_{1}(l, m)\right) \frac{t^{l}}{l !} \\
=\sum_{n=0}^{\infty} \sum_{r=0}^{n} \sum_{l=0}^{n}\left(\begin{array}{l}
n \\
r
\end{array}\right)\left(\begin{array}{l}
n \\
l
\end{array}\right) D_{r} F_{n-r-l}(y) \sum_{m=0}^{l} \frac{p(m+1) m !}{(m+1)^{k}} S_{1}(l, m) \frac{t^{n}}{n !}
\end{gathered}
$$

Therefore, by comparing the coefficients on both sides of (3.9), we obtain the following theorem.

Theorem 3.3. For $n \geq 0$ and $k \in \mathbb{Z}$, we have

$$
F_{n, p}^{(k)}=\sum_{r=0}^{n} \sum_{l=0}^{n}\left(\begin{array}{l}
n \\
r
\end{array}\right)\left(\begin{array}{l}
n \\
l
\end{array}\right) D_{r} F_{n-r-l}(y) \sum_{m=0}^{l} \frac{p(m+1) m !}{(m+1)^{k}} S_{1}(l, m) .
$$

We observe that

$$
\begin{aligned}
\sum_{n=0}^{\infty} \frac{d}{d x} F_{n, p}^{(k)}(x ; y) \frac{t^{n}}{n !} & =\frac{1}{t\left(1-y\left(e^{t}-1\right)\right)} u_{k}(\log (1+t) \mid p) \frac{d}{d x} e^{x t} \\
& =\sum_{n=0}^{\infty} F_{n, p}^{(k)}(x ; y) \frac{t^{n+1}}{n !}
\end{aligned}
$$

Therefore, we give the following theorem.

Theorem 3.4. For $n \geq 0$ and $k \in \mathbb{Z}$, we have

$$
\frac{d}{d x} F_{n, p}^{(k)}(x ; y)=n F_{n-1, p}^{(k)}(x ; y) .
$$

By theorem 3.4, we consider that

$$
\begin{gathered}
\int_{\alpha}^{\beta} F_{n, p}^{(k)}(x ; y) d x=\frac{1}{n+1} \int_{\alpha}^{\beta} \frac{d}{d x} F_{n, p}^{(k)}(x ; y) d x \\
=\frac{F_{n+1, p}^{(k)}(\beta ; y)-F_{n+1, p}^{(k)}(\alpha ; y)}{n+1}
\end{gathered}
$$


Thus, we provide the following theorem.

Theorem 3.5. For $n \geq 0$ and $k \in \mathbb{Z}$, we have

$$
\int_{\alpha}^{\beta} F_{n, p}^{(k)}(x ; y) d x=\frac{F_{n+1, p}^{(k)}(\beta ; y)-F_{n+1, p}^{(k)}(\alpha ; y)}{n+1} .
$$

and

$$
\int_{\alpha}^{\beta} F_{n, p}^{(k)}(x ; y) d y=\frac{F_{n+1, p}^{(k)}(x ; \beta)-F_{n+1, p}^{(k)}(x ; \alpha)}{n+1} .
$$

\section{Conclusion}

Motivated by the definition of the type 2 poly-Bernoulli introduced by Kim-Kim [9], in the present paper, we have considered a class of new generating function for the poly-Fubini polynomials, called the type 2 poly-Fubini polynomials, by means of the polyexponential function. Then, we have derived some useful relations and properties. We have showed that the type 2 poly-Fubini polynomials equal a linear combination of the classical Fubini polynomials and Stirlings numbers of the first kind. In a special case, we have given a relation between the type 2 poly-Fubini polynomials and Bernoulli polynomials of order $r$. Moreover, by inspired by definition of the unipoly-Bernoulli polynomials introduced by Kim-Kim [9], we have introduced the unipoly-Fubini polynomials by means of unipoly function and have given multifarious properties including derivative and integral properties. Moreover, we have provided a correlation between the unipoly-Fubini polynomials and the classical Fubini polynomials.

Acknowledgements: No.

\section{References}

[1] Boyadzhiev, K. N. A series transformation formula and related polynomials. Int. J. Math. Math. Sci. 2005, 23,3849-3866.

[2] Dil, A.; Kurt, V. Investing geometric and exponential polynomials with EulerSeidel matrices. J. Integer Sequences. 2011, 14,1-12.

[3] Duran, U.; Acikgoz, M. Truncated Fubini polynomials. Mathematics. 2019, 7, 431; doi:10.3390/math7050431.

[4] Dolgy, D.V.; Jang, L.C.; A note on the polyexponential Genocchi polynomials and numbers. Symmetry. 2020, 12,1007; doi:10.3390/sym12061007.

[5] Guohui, C,; Li, C. Some Identities Involving the Fubini Polynomials and Euler Polynomials, Mathematics. 2018, 6,300; doi:10.3390/math6120300.

[6] Khan, W.A.; Nisar, K.S.; Duran, U.; Acikgoz, M.; Araci, S. Multifarious implicit summation formulae of Hermite-based poly-Daehee polynomials. Appl. Math. Inf. Sci. 2018, 12(2), 305-310.

[7] Khan, W.A.; Nisar, K.S.; Baleanu, D. A note on $(p, q)$-analogue type of Fubini numbers and polynomials. AIMS Math. 2020, 5(3), 2743-2757.

[8] Kim, D.S.; Kim, T. Higher-order Bernoulli and poly-Bernoulli mixed type polynomials. Georgian Math. J. 2015, 22, 265-272.

[9] Kim, D.S.; Kim, T. A note on polyexponential and unipoly functions. Russ. J. Math. Phys. bf 2019, 26(1), 40-49.

[10] Kim, T. A note on degenerate Stirling polynomials of the second kind. Proc. Jangjeon Math. Soc. 2017, 20(3), 319-331. 
[11] Kim, T.; Jang,L.-C.; Kim, D.S.; Kim, H.-Y. Some identities on type 2 degenerate Bernoulli polynomials of the second kind. Symmetry. 2020, 12(4), Article ID 510(2020).

[12] Kim, T.; Kim, D.S. Degenerate Laplace transform and degenerate gamma function. Russ. J. Math. Phys. 2017, 24(2), 241-248.

[13] Kim, T.; Khan, W.A.; Sharma, S. K.; Ghayasuddin, M. A note on parametric kinds of the degenerate poly-Bernoulli and poly-Genocchi polynomials. Symmetry. 2020, 12(4), Article ID 614.

[14] Kim, T.; Kim, D. S.; Dolgy, D. V.; Kwon, J.; Some identities on generalized degenerate Genocchi and Euler numbers. Informatica. 2020, 31(4), 42-51.

[15] Kim, D.S.; Kim, T. A note on type 2 Changhee and Daehee polynomials. arXiv:1809.05217v1 [math.NT] 14 Sep 2018.

[16] Kim, D.S.; Kim, T.; Kwon, H.-I.; Park, J.-W. Two variable higher-order Fubini polynomials. J. Korean Math. Soc. 2018, 55(4), 975-986.

[17] Kim, T.; Kim, D.S.; Jang, G.-W. A note on degenerate Fubini polynomials. Proc. Jangjeon Math. Soc. 2017, 20(4), 521-531.

[18] Kim, D.S.; Jang, G.-W.; Kwon, H.-I.; Kim, T. Two variable higher-order degenerate Fubini polynomials. Proc. Jangjeon Math. Soc. 2018, 21(1), $5-22$.

[19] Kim, T.; Kim, D.S.; Kwon, J.; Lee, H. Degenerate polyexpnential functions and type 2 degenerate poly-Bernoulli numbers and polynomials. Adv. Differ. Equ. 2020, 2020:168, 12pp.

[20] Kim, D.S.; Kim, T. A note on a new type of degenerate Bernoulli numbers. Russ. J. Math. Phys. 2020, 27(2), 227-235.

[21] Kim, T.; Kim, D.S.; Kwon, J.; Kim, H.Y. A note on degenerate Genocchi and poly-Genocchi numbers and polynomials. J. Ineq. Appl. 2020, 2020:110, $13 p p$.

[22] Kim, T.; Kim, D.S. Degenerate polyexponential functions and degenerate Bell polynomials. J. Math. Anal. Appl. 2020, 487(2), 124017.

[23] Kargin, L. Some formulae for products of Fubini polynomials with applications. 2016, arXiv:1701.01023v1[math.CA] 23 Dec 2016.

[24] Kilar, N.; Simsek, Y. A new family of Fubini type numbers and polynomials associated with Apostol-Bernoulli numbers and polynomials. J. Korean Math. Soc. 2017, 54(2), 1605-1621.

[25] Kilar, N.; Simsek, Some relationships between Fubini type polynomials and other special numbers and polynomials. AIP Conference Proc. 2019, 2116(1),doi.org/10.1063

[26] Lewin, L. Polylogarithms and Associated Functions; With a Foreword by A.J. Vander Poorten; North-Holland Publishing Co.: Amsterdam, NY, USA, 1981.

[27] Lee, D.S.; Kim, H.-Y.; Jang, L.-C. Type 2 degenerate poly-Euler polynomials. Symmetry. 2020, 12, Article ID 1011.

[28] Sharma, S. K.; Khan, W. A.; Ryoo, C. S. A parametric kind of the degenerate Fubini numbers and polynomials. Mathematics. 2020, 8,405; doi:10.3390/math8030405.

[29] Sharma, S. K.; Khan, W. A.; Ryoo, C. S. A parametric kind of Fubini polynomials of a complex variable. Mathematics. 2020, 8,643; doi:10.3390/math8040643./1.5114093.

[30] Tanny, S. M. On some numbers related to Bell numbers. Canad. Math. Bull. 1974, $17,733-738$. 